\title{
Perceived Stress and Associated Factors Among Pregnant Women Attending Antenatal Care in Urban Thailand
}

This article was published in the following Dove Press journal: Psychology Research and Behavior Management

\author{
Wassapol Thongsomboon' \\ Kasemsis Kaewkiattikun (D) \\ Nitchawan Kerdcharoen ${ }^{2}$ \\ 'Department of Obstetrics \& \\ Gynecology, Faculty of Medicine Vajira \\ Hospital, Navamindradhiraj University, \\ Bangkok, Thailand; ${ }^{2}$ Department of \\ Psychiatry, Faculty of Medicine Vajira \\ Hospital, Navamindradhiraj University, \\ Bangkok, Thailand
}

Background: Perceived stress during pregnancy is associated with adverse obstetric outcomes. Antenatal perceived stress is still unaware and under-diagnosed during routine antenatal care. There has not yet been a study of prevalence and associated factors among pregnant women living in urban areas in Thailand. Understanding antenatal perceived stress is important to improve maternal and neonatal outcomes.

Objective: To identify the prevalence, associated factors, and predictive factors of perceived stress in pregnant women living in an urban area.

Materials and Methods: This cross-sectional study was conducted from December 1, 2019 to February 29, 2020 among pregnant women attending antenatal care at the Department of Obstetrics and Gynecology, Faculty of Medicine Vajira Hospital, Bangkok, Thailand. The participants were interviewed using a structured questionnaire which included demographic data, obstetric data, serious life event data, and a Thai language version of the 10-item Perceived Stress Scale (T-PSS-10) to assess perceived stress symptoms.

Results: Of a total 403 pregnant women, the prevalence of perceived stress symptoms in antenatal pregnant women was $23.6 \%$. Perceived stress symptoms were significantly associated with divorce $(\mathrm{p}=0.001)$, separation from spouse $(\mathrm{p}=0.005)$, physical or psychological trauma from family $(p=0.005)$, marital conflict $(p<0.001)$, and family conflict $(p<0.001)$. Results from multiple logistic regression found that significant predictive factors for perceived stress symptoms in pregnant women were marital conflict (AOR 3.10, 95\% CI 1.74-5.52, $\mathrm{p}<0.001$ ) and family conflict (AOR 3.24, 95\% CI 1.59-6.60, $\mathrm{p}=0.001$ ).

Conclusion: This study demonstrated that the prevalence of perceived stress symptoms in pregnant women living in an urban area in Thailand was $23.6 \%$. Perceived stress symptoms were significantly associated with divorce, separation from spouse, physical or psychological trauma from family, marital conflict, and family conflict. Predictive factors for perceived stress symptoms were marital conflict and family conflict.

Keywords: perceived stress, pregnancy, antenatal perceived stress, PSS-10, prevalence, urban, Thailand

\section{Introduction}

Pregnancy is recognized as a stressful event in a woman's life that needs enormous psychological adjustment. During pregnancy, the hormonal responses of both the hypothalamic pituitary adrenal (HPA) axis and the sympathetic nervous system to emotional and physical stressors are severely attenuated during pregnancy. ${ }^{1}$ Moreover, pregnant women will encounter mixed feelings of happiness, insecurity, and fear that lead to a great desire for support from their partner and/or family. The
Correspondence: Kasemsis

Kaewkiattikun

Department of Obstetrics \& Gynecology,

Faculty of Medicine Vajira Hospital,

Navamindradhiraj University, Bangkok

10300, Thailand

Tel +668 9536660l

Email kasem38@hotmail.com
Psychology Research and Behavior Management 2020:13 III5-II22

1 | 5

DovePress

I

in 口 
interrelation of these factors leads to an imbalance between allostatic stress, the demands of life, and individual adaptive capacity that pregnant women perceive as stress. ${ }^{1}$

Perceived stress is defined as the feelings or thoughts that an individual has about how much a stress event or situation occurs at a given time or over a given period. ${ }^{2}$ Antenatal perceived stress during pregnancy is common, with $78 \%$ experiencing low to moderate and $6 \%$ high stress. ${ }^{3}$ Prevalence of perceived stress in pregnancy varies from $11.6 \%$ to $46.7 \%{ }^{4-7}$ However, there have been few worldwide reports of prevalence of antenatal perceived stress.

Perceived stress during pregnancy is clearly known to be associated with poor obstetric outcomes, including preterm birth, preterm delivery and low birthweight. ${ }^{8-11}$ Moreover, maternal stress during pregnancy can affect iron transfer and the iron status of infants at delivery, resulting in lower iron stores at birth, low erythrocyte iron, increasing the likelihood of Stage 1 iron deficiency at 1 year of age. ${ }^{12}$ In addition, perceived stress also has a strong negative impact on the quality of life of pregnant women. $^{13}$

There have been few reports of associated factors of antenatal perceived stress despite the high prevalence and negative effects on maternal and neonatal health. Previous reports of factors associated with perceived stress were varied and inconclusive, such as gravida, gestational age, family income, pregnancy intention, husband's employment status, medical co-morbidities, and family support. $^{3-7,14,15}$ The variety of these factors depends on population, sample size, stress-screening tool, and cultural context.

Although there is ample research that links stress and pregnancy, little is known about the perception of stress among pregnant women living in an urban area. Generally, those living in urban areas are confronted with more stressors and perceived greater overall stress than those in rural areas, ${ }^{16}$ even though socioeconomic conditions, infrastructure, and healthcare facilities are clearly better than in rural areas. Higher urban stress may be caused by many factors, such as complexity and crowding, pollution, family and social stress, occupational and economic strains, a less controllable environment, neighborhood problems, and disturbance of chronobiological rhythms. ${ }^{17}$ There have been few studies on antenatal perceived stress, especially in urban areas. Thus, this study explored perceived stress symptoms among pregnant women living in an urban area.

To our knowledge, no review of the literature has reported the prevalence and associated factors of perceived stress among pregnant women in urban Thailand and in Southeast Asian region. Therefore, this study fills the gap in knowledge of antenatal perceived stress in these settings. Our study aimed to explore the prevalence of perceived stress symptoms and associated factors in pregnant women attending antenatal care in an urban area in Thailand. Understanding antenatal perceived stress is important for institutions in order to create strategies and guidelines for treating maternal stress. The results of this study provide further information for management of maternal perceived stress to prevent adverse maternal and neonatal outcomes.

\section{Materials and Methods}

A cross-sectional research study was conducted from December 1, 2019 to February 29, 2020 at the Department of Obstetrics and Gynecology, Faculty of Medicine Vajira Hospital, Navamindradhiraj University, Bangkok, Thailand. The research protocol was approved by the Institutional Review Board (IRB) and Ethics Committee of the Faculty of Medicine Vajira Hospital. In addition, this study was conducted as stated in the Declaration of Helsinki.

The population consisted of all pregnant women who attended antenatal care clinic at the Department of Obstetrics \& Gynecology, Faculty of Medicine Vajira Hospital. Inclusion criteria were low-risk pregnant women of Thai nationality who could communicate in Thai and who had been living in Bangkok for at least 1 year. Exclusion criteria were conditions that may affect emotional state or antenatal perceived stress at the time of interview such as a history of psychiatric illness (depressive disorder, schizophrenia, anxiety disorder), intellectual disability, coexisting medical diseases (overt diabetes mellitus, hypertension, thyroid diseases, cardio-vascular diseases, oncologic diseases), coexisting gynecologic diseases (gynecologic neoplasm, uterine anomalies, infertility), current pregnancy complications (antepartum hemorrhage, pre-eclampsia, cardio-vascular diseases, gestational diabetes, multifetal pregnancy, recurrent abortion, preterm labor, premature rupture of membranes), and fetal complications (non-reassuring fetal heart rate, fetal distress, fetal anomalies, intrauterine growth retardation, oligohydramnios) 
Sample size was calculated based on a previous study in Saudi Arabia, in which the study setting, and perceived stress measurement tool were the same as this study. They found that the prevalence of perceived stress in pregnant women was $33.4 \% .^{5}$ The powers of $80 \%$ and a level of confidence of $95 \%$ were applied to determine the difference between groups. By adding $15 \%$ for incomplete data, the calculated sample size was at least 403 samples. Computerized simple random sampling technique was used.

All participants were informed about all relevant aspects of the study, including its aim, interview procedures, anticipated benefits, and potential hazards at an antenatal clinic by a well-trained research assistant. Confidentiality of the data was strictly maintained. The research assistant would provide all participants adequate time to make a decision whether to participate voluntarily and independently. The non-participants would receive standard care without affecting the quality of care they received. Their right to deny participating in the study at any time was also ensured. All participants gave informed consent after understanding all study processes. Participants, under the legal age of consent (under 19 years old), and their parents or legal guardians were informed of the study processes by a well-trained research assistant at the antenatal clinic. After understanding all processes, their parents or legal guardians provided informed consent on their behalf to the research assistant.

Eligible participants were interviewed by a well-trained research assistant, using a structured questionnaire. The questionnaire included demographic data (age, body mass index, marital status, educational status, occupation, husband's occupation, family income, smoking, and alcohol drinking); obstetrics data (pregnancy intention, parity, gestational age, and previous abortion); and serious life event data (death of close relatives, separation from spouse, fired/forced to change job, physical/psychological trauma from family, suicidal idea/suicidal attempts, marital conflict, and family conflict).

The questionnaire of maternal perceived stress was assessed by the Perceived Stress Scale (PSS), which is the most widely used psychological instrument for measuring the perception of stress. The PSS was originally developed by Cohen et al in $1983 .{ }^{18}$ The PSS is a brief and easy-to- administer self-assessment tool to assess the degree to which situations in one's life are appraised as stressful in the preceding month that are still affecting respondents' stress levels. The PSS used in this study was a 10-item version (PSS10) which was more appropriate for measuring perceived stress in pregnant women than the original 14-item version (PSS-14). ${ }^{19}$ The PSS has been translated into various languages. The Thai version of the PSS-10 (T-PSS-10) was assessed by studying in 479 adult participants in Northern Thailand. The study concluded that the T-PSS-10 had excellent goodness-of-fit, good reliability, and high validity for assessing the stress perception level within a Thai cultural context. ${ }^{20}$ The T-PSS10 is a 5-point Likert-type scale ranging from 0 (never) to 4 (very often), representing how often participants had perceived stress symptoms within the past month. The sum of all 10 items was calculated for the T-PSS-10 score. Assessment scores ranged from 0 to 40, with higher scores indicating greater perceived stress symptoms. Scores of 21-40 were considered to reveal the presence of perceived stress symptoms. ${ }^{18}$ Pregnant women who had a positive screening test were referred to a psychiatrist for re-evaluation and proper management.

The primary outcome of this study was identifying the prevalence of perceived stress symptoms among pregnant women living in an urban area. The secondary outcomes were exploring associated factors and predictive factors of perceived stress symptoms during pregnancy. The data were analyzed by a statistician using SPSS version 22 (IBM Corp., Armonk, NY, USA). ${ }^{21}$ Student's $t$-test and Chi-squared test were used for data analysis. Univariate and multivariate analyses were further entered into the logistic regression analysis to determine independent predictors of perceived stress in pregnant women and presented as odds ratios and 95\% CI. The analysis results were considered statistically significant if the P-value was $<0.05$.

\section{Result}

During the study period, 415 pregnant women who fulfilled the inclusion and exclusion criteria were approached, 10 denied participating in the study and 2 did not completely respond to the questionnaire. A total of 403 pregnant women completing the results were eligible for the study. Table 1 shows the prevalence of perceived stress symptoms in pregnant women was $23.6 \%$. The mean age of participants was $27.91 \pm 6.38$ years with age range between 13 and 43 years old. The demographic characteristics showed that most of the participants were adult pregnancy $(90.8 \%)$, had normal body mass index $(50.7 \%)$, were married $(76.9 \%)$, had an elementary and high school education (48.1\%), were employed (80.6\%), 
Table I Baseline Characteristics of Pregnant Women and Association with Perceived Stress Symptoms $(n=403)$

\begin{tabular}{|c|c|c|c|c|}
\hline \multirow[t]{2}{*}{ Characteristics } & \multirow{2}{*}{$\begin{array}{l}\text { Total } \\
(n=403)\end{array}$} & \multicolumn{2}{|c|}{ Perceived Stress Symptoms } & \multirow[t]{2}{*}{ p-value } \\
\hline & & Yes $(n=95)$ & No $(n=308)$ & \\
\hline Total & $403(100.0)$ & $95(23.6)$ & $308(76.4)$ & \\
\hline Age (years) (mean $\pm \mathrm{SD})$ & $27.91 \pm 6.38$ & $28.92 \pm 6.19$ & $27.60 \pm 6.41$ & 0.080 \\
\hline Adolescence (12-19 years) & $37(9.2)$ & $6(16.2)$ & $31(83.8)$ & 0.370 \\
\hline Adult (>19 years) & $366(90.8)$ & $89(24.3)$ & $277(75.7)$ & \\
\hline \multicolumn{5}{|c|}{ BMI at the beginning of pregnancy $\left(\mathrm{kg} / \mathrm{m}^{2}\right)$} \\
\hline Normal & $204(50.7)$ & $54(26.5)$ & $150(73.5)$ & 0.165 \\
\hline Overweight/obese & $199(49.3)$ & $41(20.6)$ & $158(79.4)$ & \\
\hline \multicolumn{5}{|l|}{ Marital status } \\
\hline Unmarried & $67(16.6)$ & $21(31.3)$ & $46(68.7)$ & 0.001 \\
\hline Married & $310(76.9)$ & $62(20.0)$ & $248(80.0)$ & \\
\hline Divorced & $26(6.5)$ & $12(46.2)$ & $14(53.8)$ & \\
\hline \multicolumn{5}{|l|}{ Educational status } \\
\hline Elementary and high school & $194(48.1)$ & $36(18.6)$ & $158(81.4)$ & 0.061 \\
\hline Diploma degree & $59(14.6)$ & $15(25.4)$ & $44(74.6)$ & \\
\hline Bachelor's and master's degree & $150(37.3)$ & $44(29.3)$ & $106(70.7)$ & \\
\hline \multicolumn{5}{|l|}{ Occupation } \\
\hline Unemployed or student & $78(19.4)$ & $16(20.5)$ & $62(79.5)$ & 0.511 \\
\hline Employed & $325(80.6)$ & $85(26.2)$ & $240(73.8)$ & \\
\hline \multicolumn{5}{|l|}{ Husband's occupation } \\
\hline Unemployed or student & $24(6.0)$ & $7(29.2)$ & $17(70.8)$ & 0.506 \\
\hline Employed & $379(94.0)$ & $88(23.2)$ & $291(76.8)$ & \\
\hline \multicolumn{5}{|l|}{ Family incomes (THB) } \\
\hline$<10,000$ & $32(7.9)$ & $5(15.6)$ & $27(84.4)$ & 0.290 \\
\hline $10,000-20,000$ & $86(21.3)$ & $17(19.8)$ & $69(80.2)$ & \\
\hline$>20,000$ & $285(70.8)$ & $73(25.6)$ & $212(74.4)$ & \\
\hline \multicolumn{5}{|l|}{ Smoking during pregnancy } \\
\hline No & $399(99.0)$ & $93(23.3)$ & $306(76.7)$ & 0.237 \\
\hline Yes & $4(1.0)$ & $2(50.0)$ & $2(50.0)$ & \\
\hline \multicolumn{5}{|l|}{ Alcohol drinking during pregnancy } \\
\hline No & $401(99.5)$ & $94(23.4)$ & $307(76.6)$ & 0.416 \\
\hline Yes & $2(0.5)$ & I (50.0) & I (50.0) & \\
\hline \multicolumn{5}{|l|}{ Pregnancy intention } \\
\hline No & $184(45.7)$ & $46(25.0)$ & $138(75.0)$ & 0.536 \\
\hline Yes & $219(54.3)$ & $49(22.4)$ & $170(77.6)$ & \\
\hline \multicolumn{5}{|l|}{ Parity } \\
\hline Primiparity & $156(38.7)$ & $36(23.1)$ & $120(76.9)$ & 0.852 \\
\hline Multiparity & $247(61.3)$ & $59(23.9)$ & $188(76.1)$ & \\
\hline \multicolumn{5}{|l|}{ Current gestational age } \\
\hline First trimester & $57(14.2)$ & $13(22.8)$ & $44(77.2)$ & 0.263 \\
\hline Second trimester & $167(4 \mid .4)$ & $46(27.5)$ & $12 \mid(72.5)$ & \\
\hline Third trimester & $179(44.4)$ & $36(20.1)$ & I43 (79.9) & \\
\hline \multicolumn{5}{|l|}{ Previous abortion } \\
\hline No & $347(86.1)$ & $80(23.1)$ & $267(76.9)$ & 0.542 \\
\hline Yes & $56(13.9)$ & $15(26.8)$ & $4 \mathrm{I}(73.2)$ & \\
\hline
\end{tabular}

(Continued) 
Table I (Continued).

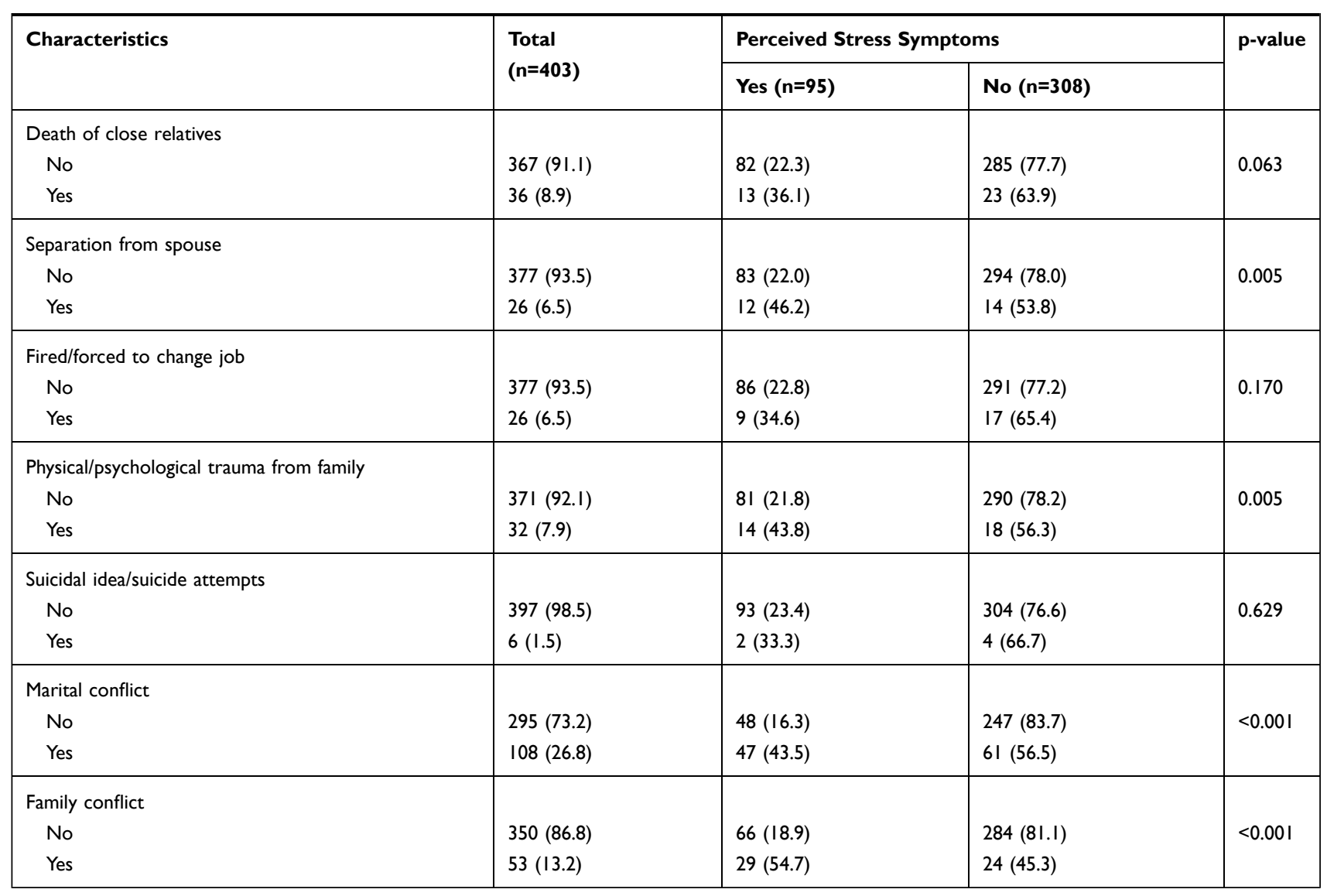

Note: Data are presented as $\mathrm{n}(\%)$.

Abbreviation: THB, Thai baht.

had high family income $(70.8 \%)$, were non-smokers (99.0\%), and drank no alcohol (99.5\%). The obstetric characteristics showed that the majority had intended pregnancy $(54.3 \%)$, were multiparity $(61.3 \%)$, were third trimester $(44.4 \%)$, and had no previous abortion (86.1\%). The survey of serious life events during pregnancy showed that most of the participants had no recent death of close relatives $(91.1 \%)$, were not separated from their spouse (93.5\%), had not been fired or forced to change job (93.5\%), had no physical or psychological trauma from family $(92.1 \%)$, had no suicidal ideation or suicide attempts $(98.5 \%)$, had no marital conflict $(73.2 \%)$, and had no family conflict $(86.8 \%)$.

The association between baseline characteristics and maternal perceived stress is shown in Table 1. Pregnant women with perceived stress were significantly more likely to be divorced ( $\mathrm{p}=0.001$ ), be separated from spouse $(\mathrm{p}=0.005)$, have experienced physical or psychological trauma from family $(\mathrm{p}=0.005)$, have marital conflict $(\mathrm{p}<0.001)$, and family conflict $(\mathrm{p}<0.001)$.
Table 2 shows the analysis of factors predicting perceived stress symptoms. The univariate logistic regression analysis found that marital status, separation from spouse, physical or psychological trauma from family, marital conflict, and family conflict were significant factors $(p<0.10)$. After adjusting OR estimated by multiple logistic regression adjusting for marital status, separation from spouse, physical or psychological trauma from family, marital conflict, and family conflict were analyzed. The adjusted OR estimated by multivariate logistic regression analysis revealed that the significant factors predicting perceived stress symptoms were marital conflict (AOR $3.10,95 \%$ CI $1.74-5.52, \mathrm{p}<0.001$ ), and family conflict (AOR 3.24, 95\% CI 1.59-6.60, $\mathrm{p}=0.001$ ).

\section{Discussion}

This cross-sectional study discovered that the prevalence of perceived stress symptoms in pregnant women living in urban Thailand was $23.6 \%$. This finding is lower than that of previous studies in urban areas. It was $33.4 \%$ in 
Table 2 Univariate and Multiple Logistic Regression Analysis of Factors Associated with Perceived Stress Symptoms

\begin{tabular}{|c|c|c|c|c|c|c|}
\hline \multirow[t]{2}{*}{ Factors } & \multicolumn{3}{|c|}{ Univariable Analysis } & \multicolumn{3}{|c|}{ Multivariable Analysis } \\
\hline & $\mathbf{O R}^{\mathbf{a}}$ & $95 \% \mathrm{Cl}$ & p-value & $\mathbf{A O R}^{\mathbf{b}}$ & $95 \% \mathrm{Cl}$ & p-value \\
\hline \multicolumn{7}{|l|}{ Marital status } \\
\hline Unmarried & 1.00 & Reference & & 1.00 & Reference & \\
\hline Married & 1.78 & $(0.99-3.18)$ & 0.053 & 1.86 & $(0.95-3.63)$ & 0.071 \\
\hline Divorced & 6.23 & $(1.97-19.67)$ & 0.002 & 2.00 & $(0.35-11.43)$ & 0.435 \\
\hline \multicolumn{7}{|c|}{ Separation from spouse } \\
\hline No & 1.00 & Reference & & 1.00 & Reference & \\
\hline Yes & 3.04 & $(1.35-6.82)$ & 0.007 & 1.31 & $(0.33-5.27)$ & 0.703 \\
\hline \multicolumn{7}{|c|}{ Physical/psychological trauma from family } \\
\hline No & 1.00 & Reference & & 1.00 & Reference & \\
\hline Yes & 2.79 & $(1.33-5.84)$ & 0.007 & 1.65 & $(0.63-4.32)$ & 0.311 \\
\hline \multicolumn{7}{|l|}{ Marital conflict } \\
\hline No & 1.00 & Reference & & 1.00 & Reference & \\
\hline Yes & 3.97 & $(2.43-6.47)$ & $<0.001$ & 3.10 & $(1.74-5.52)$ & $<0.001$ \\
\hline \multicolumn{7}{|l|}{ Family conflict } \\
\hline No & 1.00 & Reference & & 1.00 & Reference & \\
\hline Yes & 5.20 & $(2.84-9.5 I)$ & $<0.001$ & 3.24 & $(1.59-6.60)$ & 0.001 \\
\hline
\end{tabular}

Notes: ${ }^{a}$ Crude odds ratio estimated by binary logistic regression. ${ }^{b}$ Adjusted odds ratio estimated by multiple logistic regression. Variable was included in multivariable model due to $\mathrm{p}$-value $<0.050$ in univariable analysis. Model Summary: -2 Log likelihood $=365.79$, Cox and Snell's $R^{2}=0.169$, and Nagelkerke's $R^{2}=0.254$.

Abbreviations: OR, odds ratio; AOR, adjusted odds ratio; $\mathrm{Cl}$, confidence interval.

Saudi Arabia ${ }^{5}$ and $34 \%$ in Kathmandu. ${ }^{22}$ The prevalence in this study is quite low even though living in urban areas is more likely to expose to stressors than in rural areas. ${ }^{17}$ Moreover, pregnant women living in urban areas perceive higher stress over self-physical concerns, labor and delivery process, and baby's well-being than those in rural women. ${ }^{16}$ This assumption is confirmed by a study in the United State of America that explicitly found that pregnant women living in urban areas perceived greater overall stress than those living in rural areas (mean PSS 8.0-8.9 in urban vs 4.5-7.7 in rural areas). ${ }^{18}$ The possible explanation for lower prevalence in this study from other urban settings may be due to each area's cultural context and the studied participants' differences.

In Thai culture, family relations are strong and close, and there is a sense of community and social connection that creates a network of social support resulting in a reduction of perceived stress. This culture is likely to be important in reducing stress even for those living in urban areas. Therefore, low perceived stress prevalence was found in the present study. Encouraging family to give social support to pregnant women is crucial for diminishing stress. ${ }^{15}$ There is strong evidence from a prior study from Iran that pregnant women with favorable social support had significantly less stress than those with unfavorable social support $(p<0.001){ }^{23}$

Another possible reason to explain the different result of this study from other studies in urban areas is the difference among the studied participants. A recent systematic review demonstrated a significant association between increased antenatal perceived stress and primipara, high educational status, and low family income. ${ }^{24}$ The low prevalence of perceived stress in this study may be due to most pregnant women were multipara, had low education, and high family income. In contrast, higher prevalence was observed in previous studies from urban Saudi Arabia $^{5}$ and Kathmandu, ${ }^{22}$ in which pregnant women were mostly primipara, had high education, and low family income.

In this study, maternal perceived stress symptoms were significantly associated with divorced, separation from spouse, physical or psychological trauma from family, marital conflict, and family conflict. It is noteworthy that all these factors were serious life events that pregnant women perceived as stressful events. However, these associated factors remain under-recognized and under-detected during routine antenatal care. Thus, perceived stressscreening protocol should be applied in routine antenatal 
care to identify not only pregnant women experiencing stress, but also those with stress-associated factors.

After adjusting for confounding effects using multiple logistic regression analysis to adjust for significant associated factors, this study found that pregnant women with marital conflict, and family conflict were thrice as likely to have high levels of perceived stress symptoms, as compared to those without these risk factors. These events certainly increase feelings of stress. Therefore, it is necessary to inform and counsel all pregnant women about negative consequences of conflicts as soon as possible to prevent stress. As for marital and family conflict, it is extremely difficult to know about these events during routine antenatal care other than through an in-depth interview. Even though these factors are difficult to overcome, early recognition of marital and family conflict is crucial for early psychiatric support. Moreover, specific health behavior interventions to reduce stress should be provided to pregnant women and their families.

The results of this study strongly suggest all institutions should apply stress-screening protocols to existing antenatal programs and develop strategies to provide practical support to reduce the likelihood of perceived stress. Moreover, healthcare providers should discover associated risk factors and provide psychiatric counseling to all pregnant women and their families during the antenatal period to decrease stress and the risk of adverse pregnancy outcomes. Moreover, stress reduction programs are necessary to help pregnant women in urban areas develop stress management skills specific to urban social stress. ${ }^{15,17}$

This study had several strengths, including large sample size, utilization of standard stress-screening tool (PSS), and interviews were conducted by a well-trained research assistant. However, this study was limited by a crosssectional design such that the relationship between variables could not be proven. Most of the data were selfreported with no means of verification, and thus subject to bias. In addition, the measurement of factors such as marital and family relationships/social support may be subject to bias. Another limitation of this study includes factors that might have affected the answers, such as spouse and family relationships, attitudes and beliefs, and individual perceptions of stressors that were not evaluated. Further research would compare perceived stress among pregnant women in urban and rural settings. Other studies are planned to further investigate the effect of stressscreening implementation on maternal stress. Finally, suggestions for future research should be extended to study in multicenter hospitals in urban areas.

\section{Conclusion}

The prevalence of perceived stress symptoms among pregnant women living in urban Thailand was $23.6 \%$ according to The Thai version of the PSS-10 (T-PSS-10). Antenatal perceived stress symptoms were significantly associated with divorce, separation from spouse, physical or psychological trauma from family, marital conflict, and family conflict. Significant predictive factors for perceived stress symptoms were marital conflict and family conflict. Screening of perceived stress should be included in routine antenatal care.

\section{Acknowledgment}

The authors would like to acknowledge all participants in this research. We also express our gratefulness and thanks to $\mathrm{Mr}$ Jason Cullen for proofreading the manuscript. Finally, this study was accomplished with funding from Navamindradhiraj University. The funders had no role in the study process, preparation of the manuscript, and decision to publish.

\section{Disclosure}

The authors report no conflicts of interest in this work.

\section{References}

1. González-Ochoa R, Sánchez-Rodríguez EN, Chavarría A, GutiérrezOspina G, Romo-González T. Evaluating stress during pregnancy: do we have the right conceptions and the correct tools to assess it? J Pregnancy. 2018;1-20.

2. Phillips AC. Perceived Stress. In: Gellman MD, Turner JR, editors. Encyclopedia of Behavioral Medicine. New York, NY: Springer; 2013.

3. Woods SM, Melville JL, Guo Y, Fan MY, Gavin A. Psychosocial stress during pregnancy. Am J Obstet Gynecol. 2010;202(1):61.e1-61. e7. doi:10.1016/j.ajog.2009.07.041

4. Engidaw NA, Mekonnen AG, Amogne FK. Perceived stress and its associated factors among pregnant women in Bale zone Hospitals, Southeast Ethiopia: a cross-sectional study. BMC Res Notes. 2019;12 (356):1-6. doi:10.1186/s13104-019-4383-0

5. Ahmed AE, Albalawi AN, Alshehri AA, AlBlaihed RM, Alsalamah MA. Stress and its predictors in pregnant women: a study in Saudi Arabia. Psychol Res Behav Manag. 2017;10:97-102. doi:10.2147/PRBM.S131474

6. Waqas A, Zubair M, Zia S, et al. Psychosocial predictors of antenatal stress in Pakistan: perspectives from a developing country. BMC Res Notes. 2020;13(160):1-6. doi:10.1186/s13104-020-05007-3

7. Yarube IU, Sani L, Saleh MIA, Alhassan AW. Assessment of perceived stress amongst primigravidae attending an ante natal clinic in Kano, Nigeria. J Afr Ass Physiol Sci. 2018;6(1):46-51.

8. Staneva A, Bogossian F, Pritchard M, Wittkowski A. The effects of maternal depression, anxiety, and perceived stress during pregnancy on preterm birth: a systematic review. Women Birth. 2015;28(3):179-193. doi:10.1016/j.wombi.2015.02.003

9. Tanpradit K, Kaewkiattikun K. The effect of perceived stress during pregnancy on preterm birth. Int J Womens Health. 2020;12:287-293. doi:10.2147/IJWH.S239138 
10. Rondo' PHC, Ferreira RF, Nogueira F, Ribeiro MCN, Lobert H, Artes R. Maternal psychological stress and distress as predictors of low birth weight, prematurity and intrauterine growth retardation. Eur J Clin Nutr. 2003;57(2):266-272. doi:10.1038/sj.ejen.1601526

11. Lima SAM, El Dib RP, Rodrigues MRK, et al. Is the risk of low birth weight or preterm labor greater when maternal stress is experienced during pregnancy? A systematic review and meta-analysis of cohort studies. PLoS One. 2018;13(7):e0200594. doi:10.1371/journal.pone. 0200594

12. Rendina DN, Blohowiak SE, Coe CL, Kling PJ. Maternal perceived stress during pregnancy increases risk for low neonatal iron at delivery and depletion of storage iron at one year. $J$ Pediatr. 2018;200:166-173.e2. doi:10.1016/j.jpeds.2018.04.040

13. Lagadec N, Steinecker M, Kapassi A, et al. Factors influencing the quality of life of pregnant women: a systematic review. $B M C$ Pregnancy Childbirth. 2018;18(455):1-14. doi:10.1186/s12884-0182087-4

14. Vijayaselvi R, Beck MM, Abraham A, Kurian S, Regi A, Rebekah G. Risk factors for stress during antenatal period among pregnant women in tertiary care hospital of southern India. J Clin Diagn Res. 2015;9(10):1-5.

15. Basharpoor S, Heydarirad H, Daryadel SJ, Heydari F, Givi HG, Kishore J. The role of perceived stress and social support among predicting anxiety in pregnant women. $J$ Holist Nurs Midwifery. 2017;27(2):9-16. doi:10.18869/acadpub.hnmj.27.2.9

16. Zust B, Natwick L, Oldani A. Stress perception among rural and urban perinatal patients. Online J Rural Nurs Health Care. 2010;10 (1):70-79. doi:10.14574/ojrnhc.v10i1.75
17. Willie TC, Powell A, Kershaw T. Stress in the city: influence of urban social stress and violence on pregnancy and postpartum quality of life among adolescent and young mothers. J Urban Health. 2016;93(1):19-35. doi:10.1007/s11524-015-0021-x

18. Cohen S, Kamarck T, Mermelstein R. A global measure of perceived stress. J Health Soc Behav. 1983;24(4):385-396. doi:10.2307/2136 404

19. Yokokura AVCP, da Silva AAM, Fernandes JKB, et al. Perceived stress scale: confirmatory factor analysis of the PSS14 and PSS10 versions in two samples of pregnant women from the BRISA cohort. Cad Saude Publica. 2017;33(12):e00184615. doi:10.1590/0102-311x 00184615

20. Wongpakaran N, Wongpakaran T. The Thai version of the PSS-10: an Investigation of its psychometric properties. Biopsychosoc Med. 2010;4(6):1-6.

21. IBM Corp. IBM SPSS Statistics for Windows, Version 22.0. Armonk, NY: IBM Corp; 2013

22. Pantha S, Hayes B, Yadav BK, et al. Prevalence of stress among pregnant women attending antenatal care in a tertiary maternity hospital in Kathmandu. J Womens Health Care. 2014;3(05):183. doi:10.4172/2167-0420.1000183

23. Iranzad I, Bani S, Hasanpour S, Mohammadalizadeh S, Mirghafourvand M. Perceived social support and stress among pregnant women at health centers of Iran- Tabriz. J Caring Sci. 2014;3 (4):287-295.

24. Pais M, Pai MV. Stress among pregnant women: a systematic review. J Clin Diagn Res. 2018;12(5):1-4.
Psychology Research and Behavior Management

\section{Publish your work in this journal}

Psychology Research and Behavior Management is an international, peer-reviewed, open access journal focusing on the science of psychology and its application in behavior management to develop improved outcomes in the clinical, educational, sports and business arenas. Specific topics covered in the journal include: Neuroscience, memory and decision making; Behavior modification and management; Clinical

\section{Dovepress}

applications; Business and sports performance management; Social and developmental studies; Animal studies. The manuscript management system is completely online and includes a very quick and fair peer-review system, which is all easy to use. Visit http://www. dovepress.com/testimonials.php to read real quotes from published authors. 